\title{
Effectiveness of mindfulness by smartphone, for patients with chronic migraine and medication overuse during the Covid-19 emergency
}

\author{
Licia Grazzi ${ }^{1}$ - Paul Rizzoli ${ }^{2}$. Frank Andrasik ${ }^{3}$ \\ Published online: 13 August 2020 \\ (C) Fondazione Società Italiana di Neurologia 2020
}

\begin{abstract}
Note: This data is mandatory. Please provide.

Keywords Note: This data is mandatory. Please provide.
\end{abstract}

Chronic migraine, a disabling condition that affects the $2 \%$ of migraine population, is frequently associated with medication overuse that makes this condition difficult to treat. Many prior investigations have affirmed the efficacy and importance of having patients withdraw from all offending medications contributing to this condition, as well as point to the need to carefully follow patients after withdrawal to avoid relapses, minimize the chances of resuming recurrent overuse and maintain, hopefully improve, the clinical benefits of whatever concurrent therapeutic approach was provided.

Also, clinical results are enhanced when traditional therapies are combined with behavioral approaches, in particular mindfulness that help patients become more conscious about their symptoms and learn techniques for managing pain without medications [1]. At the Besta Institute, we have been following a cohort of patients who have completed a supervised medication withdrawal, which is followed by a specific prophylaxis for migraine, but also a specially designed program for mindfulness practice, that is administered for 6 weekly-45min sessions to patients in small groups. As the coronavirus

Licia Grazzi

licia.grazzi@istituto-besta.it

1 Headache Center, Neuroalgology Department, IRCCS Foundation "Carlo Besta" Neurological Institute, Milan, Italy

2 John Graham Headache Center, Faulkner \& Brigham Hospital, Harvard Medical School, Boston, MA, USA

3 Department of Psychology, University of Memphis, Memphis, TN, USA began to spread rapidly throughout Italy, our in-clinic approach was no longer advisable or feasible, as patients were unable to attend sessions on a regular basis. Thus, our aim in this study was to investigate the feasibility and the effectiveness of mindfulness by smart phone combined to video calls for clinical evaluation and follow-up of patients with chronic migraine and medication overuse after withdrawal program and to confirm the effectiveness after 1 year

We organized and evaluated a pilot intervention capable of using existing technology, in this case smartphones, to ensure that patients were able to receive and practice the mindfulness treatment as an adjunct to their care once they were no longer able to return to the hospital for inperson treatment. Twenty patients recruited and treated at the Neuroalgology Unit of the IRCCS Foundation "Carlo Besta" Neurological Institute, completed our standard medication withdrawal program. After that, they were provided the additional training needed for mindfulness on their smartphone practicing each day for $12 \mathrm{~min}$. All remote sessions were recorded by the expert who generally manages face-to-face sessions at the hospital. A separate weekly video call was made in order to evaluate their clinical condition and encourage and reinforce use of the pain management strategies being provided. Patients had to record their headache episodes on the daily headache diary, and they will be followed with regular meetings every 3 months up to 1 year. Fifteen patients have completed the treatment protocol to date, reporting a mean reduction of days of migraine per month of $50 \%$, with a concurrent mean reduction of medication intake per month of the same amount $-50 \%$ recorded by the daily headache diary. At the end of the program, patients 
reported migraine without aura at high frequency; they did not report medication overuse. Our preliminary findings, recorded after treatment, suggest this combination treatment modality, which allowed patients to continue their therapeutic process and be followed regularly, but remotely, after withdrawal, revealed a significant decrease in terms of clinical indexes. Adherence to the treatment was judged as high based of response that were recorded, but admittedly not evaluated systematically. Our preliminary findings confirm that this type of combined treatment (in-clinic followed by smartphone delivery outside of the clinic) can yield results similar to those we obtained in previous studies relying on in-clinic treatment alone. This approach, which evolved in response to a severe health crisis, warrants further, more controlled investigations to document and replicate both the clinical effectiveness as well as the saving in terms of time on the part of patients and staff and need for resources. The literature of the last decades $[2,3]$ promote the use of smart phone or telemedicine for clinical and therapeutic applications with encouraging results. This modality, applied during this specific and dramatic situation, allowed our patients to continue their therapeutic process reinforcing their efforts and to be followed regularly during the 1 year after withdrawal.

\section{Compliance with ethical standards}

Conflict of interest The author declares that there is no conflict of interest.

Ethical standards This article does not contain any study with human subjects performed by any of the authors.

\section{References}

1. Grazzi L, Raggi A, D'Amico D, Sansone E, Leonardi M, Andrasik F, Gucciardi A, Guido D, D'Andrea G (2018) A prospective pilot study of the effect on catecholamines of mindfulness training vs pharmacological prophylaxis in patients with chronic migraine and medication overuse headache. Cephalalgia 13:333102418801584333102418801664. https://doi.org/10.1177/0333102418801584

2. Alexander J, Joshi GP (2016) Smartphone applications for chronic pain management: a critical appraisal. J Pain Res 9:731-734

3. Adams C, Deeker-Van Weering MGH, Van Etten-Jamaludin FS, Stuiver M (2018) The effectiveness of exercise-based telemedicine on pain, physical activity and quality of life in the treatment of chronic pain: a systematic review. J Telemed Telecare 24(8):511526

Publisher's note Springer Nature remains neutral with regard to jurisdictional claims in published maps and institutional affiliations. 